\title{
Extracellular vesicles released by J774A.I macrophages reduce the bacterial load in macrophages and in an experimental mouse model of tuberculosis
}

This article was published in the following Dove Press journal:

International Journal of Nanomedicine

\begin{abstract}
Mariano García-Martínez, 1,2 Luis Vázquez-Flores, ${ }^{1,2}$ Violeta D Álvarez-Jiménez, ${ }^{1,3}$ Jessica Castañeda-Casimiro,' Miguel lbáñez-Hernández, ${ }^{4}$ Luvia Enid Sánchez-Torres,' Jorge BarriosPayán, ${ }^{5}$ Dulce Mata-Espinosa, ${ }^{5}$ Sergio Estrada-Parra,' Rommel Chacón-Salinas,' Jeanet SerafínLópez, ' Isabel Wong-Baeza,' Rogelio Hernández-Pando, ${ }^{5}$ Iris Estrada-García'

'Departamento de Inmunología, Escuela Nacional de Ciencias Biológicas (ENCB), Instituto Politécnico Nacional (IPN), Mexico City, Mexico; ${ }^{2}$ Posgrado en Inmunología, Escuela Nacional de Ciencias Biológicas (ENCB), Instituto Politécnico Nacional (IPN), Mexico City, Mexico; ${ }^{3}$ Laboratorio de Biología Molecular y Bioseguridad Nivel 3, Centro Médico Naval (CEMENAV), Secretaría de Marina Armada de México (Semar), Mexico City, Mexico; ${ }^{4}$ Departamento de Bioquímica, Escuela Nacional de Ciencias Biológicas (ENCB), Instituto Politécnico Nacional (IPN) Mexico City, Mexico; ${ }^{5}$ Sección de Patología Experimental, Departamento de Patología, Instituto Nacional de Ciencias Médicas y Nutrición Salvador Zubirán, Mexico City, Mexico
\end{abstract}

Correspondence: Iris Estrada-García Departamento de Inmunología, Escuela Nacional de Ciencias Biológicas (ENCB), Instituto Politécnico Nacional (IPN), Prolongación de Carpio y Plan de Ayala s/n, Col. Santo Tomás, Ciudad de México I 1340, México

Tel $+525557296000 \times 62507$

$\mathrm{Fax}+525557296000 \times 62369$

Email iestrada5@hotmail.com
Background: Tuberculosis is the leading cause of death by an infectious microorganism worldwide. Conventional treatment lasts at least six months and has adverse effects; therefore, it is important to find therapeutic alternatives that reduce the bacterial load and may reduce the treatment duration. The immune response against tuberculosis can be modulated by several mechanisms, including extracellular vesicles (EVs), which are nano-sized membrane-bound structures that constitute an efficient communication mechanism among immune cells.

Methods: The EVs released by the J774A.1 mouse macrophage cell line, both spontaneously (S-EV) and after infection with Mycobacterium tuberculosis H37Rv (Mtb-EV), were purified by ultra-centrifugation and size-exclusion chromatography. The size distribution and chemical composition of these EVs were evaluated, and their effect on the bacterial load and the production of cytokines was determined in both in vitro and in vivo models of $M$. tuberculosis infection.

Results: Mtb-EV are larger than S-EV, they contain M. tuberculosis-specific antigens (not detected in EVs released from M. fortuitum-infected J774A.1 cells) and are rich in phosphatidylserine, present in their outer membrane layer. S-EV, but not Mtb-EV, reduced the bacterial load and the production of MCP-1 and TNF- $\alpha$ in M. tuberculosis-infected macrophages, and these effects were reversed when phosphatidylserine was blocked with annexin V. Both S-EV and Mtb-EV significantly reduced the lung bacterial load in mice infected with M. tuberculosis after 60 days of treatment, but they had no effect on survival or on the lung pneumonic area of these mice.

Conclusion: J774A.1 macrophages infected with M. tuberculosis H37Rv released EVs that differed in size and phosphatidylserine content from spontaneously released EVs, and these EVs also had different biological effects: S-EV reduced the mycobacterial load and the cytokine production in vitro (through a phosphatidylserine-dependent mechanism), while both EVs reduced the lung bacterial load in vivo. These results are the basis for further experiments to evaluate whether EVs improve the efficiency of the conventional treatment for tuberculosis.

Keywords: tuberculosis, extracellular vesicles, phosphatidylserine, macrophages, lung disease

\section{Introduction}

Tuberculosis is the leading cause of death by an infectious microorganism worldwide. In 2017, 10 million individuals had active tuberculosis and 1.6 millions died from it. The pharmacological treatment lasts 6 months for drug-susceptible 
tuberculosis and up to 24 months for multi-drug-resistant tuberculosis, ${ }^{1}$ and the treatment causes several adverse effects, including hepatotoxicity. ${ }^{2}$ Mycobacterium tuberculosis is the main bacteria that causes this disease. ${ }^{3}$ Mycobacteria are present in the microaerosols that are expelled by patients with active pulmonary tuberculosis when they cough. When the mycobacteria reach the lungs upon inhalation, they are phagocytosed by alveolar macrophages but resist their bactericidal mechanisms, and thus persist inside these innate immune cells. Dendritic cells transport mycobacterial antigens to the draining lymph nodes and drive the activation of antigen-specific $T$ cells, which return to the lung and promote the containment of the mycobacteria inside granulomas. Granulomas are specialized structures formed by macrophages, lymphocytes, fibroblasts and other immune cells, ${ }^{4}$ and their integrity is dependent on cellular communication through the cytokines TNF- $\alpha^{5}$ and IFN- $-{ }^{6}{ }^{6}$

There is another cellular communication mechanism that has received attention in recent years: through extracellular vesicles (EVs), nano-sized spherical structures (with diameters less than $1000 \mathrm{~nm}$ ), surrounded by a bilayer lipid membrane. EVs derived from multivesicular bodies are called exosomes, while EVs derived from the cellular membrane are called ectosomes. ${ }^{7}$ In addition to lipids, EVs contain proteins and nucleic acids within them. ${ }^{8-10}$ EVs are produced by non-activated cells, ${ }^{10}$ but many activation signals increase their production and alter their composition and consequently alter the effects of these vesicles on their target cells. ${ }^{11}$ The EVs that are released by $M$. tuberculosis-infected macrophages contain mycobacterial proteins, ${ }^{12}$ induce the production of cytokines and chemokines, ${ }^{13}$ induce macrophage transmigration in vitro and macrophages recruitment in vivo, ${ }^{14}$ modify the response of other immune cells to cytokines ${ }^{15}$ and are an important source of antigens for the activation of M. tuberculosis-specific CD4 T cells. ${ }^{16}$ These characteristics suggest that EVs released by $M$. tuberculosisinfected macrophages could be useful for the treatment of tuberculosis. The low toxicity and high biological barrier permeability of EVs makes them attractive candidates as therapeutic agents, ${ }^{17,18}$ but the EVs released by $M$. tuberculosis-infected macrophages have not yet been tested as possible therapeutic agents for the treatment of tuberculosis.

In a previous study, we demonstrated that EVs released by $M$. tuberculosis-infected human neutrophils increase mycobacterial clearance in human macrophages. ${ }^{19}$ In this study, we purified and characterized the EVs released from mouse macrophages, both spontaneously and after infection with M. tuberculosis, and we tested the effects of these EVs on the bacterial load in an in vitro model of M. tuberculosis infection and in a mouse model of progressive pulmonary tuberculosis. We found that in both models, the EVs decreased the bacterial load. An auxiliary treatment that decreases the bacterial load in vivo could therefore be used to shorten the duration of the conventional anti-tuberculosis pharmacologic treatment.

\section{Materials and methods}

\section{Ethics statement}

All our protocols for animal care and use were reviewed and approved by the Bioethics Committee of ENCB-IPN (CEI-ENCB 009/2013) or by the Committee for Animal Research of the Instituto Nacional de Ciencias Médicas y Nutrición Salvador Zubirán (PAT-1825-16/19-2). All the animal experiments were carried out according to NOM 062-200-1999 guidelines of the Mexican Constitution.

\section{Mycobacterium tuberculosis culture}

M. tuberculosis H37Rv (strain TMC 102) was cultured in Middlebrook 7H9 medium (Becton Dickinson, Sparks, MD) with $10 \%$ oleic, albumin, dextrose and catalase (OADC) growth supplement (Becton Dickinson) at $37{ }^{\circ} \mathrm{C}$ for 4 weeks. Bacteria were harvested by centrifugation and stored in DMEM (Gibco, Carlsbad, CA) with $40 \%$ fetal calf serum (Gibco) at $-70{ }^{\circ} \mathrm{C}$.

\section{Mycobacterium fortuitum culture}

M. fortuitum (strain ATCC 6841) was cultured in Middlebrook medium (Becton Dickinson) with $10 \%$ oleic, albumin, dextrose and catalase (OADC) growth supplement (Becton Dickinson) at $37^{\circ} \mathrm{C}$ for $16 \mathrm{~h}$. Bacteria were harvested by centrifugation and stored in DMEM (Gibco) with $40 \%$ fetal calf serum (Gibco) at $-70{ }^{\circ} \mathrm{C}$.

\section{Purification of the EVs released by J774A.I macrophages}

The J774A.1 mouse macrophage cell line (ATCC, Manassas, VA) was cultured to near $100 \%$ confluence in $150 \mathrm{~cm}^{2}$ culture flasks, at $37{ }^{\circ} \mathrm{C}$ and $5 \% \mathrm{CO}_{2}$, in DMEM (Gibco) with $10 \%$ fetal calf serum (Gibco). Cells were washed three times with Hanks' balanced salt solution (Gibco), and the medium was replaced with $12.8 \mathrm{ml}$ of DMEM with $2.6 \%$ of EV-reduced fetal calf serum. To 
prepare this serum, $12.5 \mathrm{ml}$ of fetal calf serum were centrifuged at 160,000 $\mathrm{xg}$ in an Optima XL-90 ultracentrifuge (Beckman Coulter, Brea, CA) with a SW40Ti rotor for $3 \mathrm{~h}$ at $4{ }^{\circ} \mathrm{C}$; the upper three-fourths of the serum volume were recovered. After $4 \mathrm{~h}$ at $37{ }^{\circ} \mathrm{C}$ and $5 \% \mathrm{CO}_{2}$, the spontaneously released extracellular vesicles (S-EV) were recovered from the culture supernatants. In some cases, J774A.1 macrophages were infected with M. tuberculosis or with M. fortuitum at a MOI of 10 . Before infection, the mycobacteria were washed with Hanks' balanced salt solution (Gibco) and harvested by centrifugation. After $4 \mathrm{~h}$ at $37{ }^{\circ} \mathrm{C}$ and $5 \% \mathrm{CO}_{2}$, the extracellular vesicles released by J774A.1 cells in response to $M$. tuberculosis (Mtb-EV) or M. fortuitum (Mf-EV) infection were recovered from the culture supernatants.

Culture supernatants were collected and centrifuged at $10,000 \mathrm{xg}$ for $30 \mathrm{~min}$ to remove cells and cell debris, and at $160,000 \mathrm{xg}$ for $90 \mathrm{~min}$ to concentrate the EVs in a final volume of $1.5 \mathrm{ml}$. The EVs were purified by size-exclusion chromatography in a Sepharose 4B column (GE Healthcare, Uppsala, Sweden); EVs were eluted with endotoxin-free sterile saline solution, and $1 \mathrm{ml}$ fractions were collected. The fractions that contained EVs were identified by their absorbance at $230 \mathrm{~nm}$, which has a higher sensitivity than their absorbance at $280 \mathrm{~nm},{ }^{20}$ and EVs were stored at $4{ }^{\circ} \mathrm{C}$ for no more than one week before use (Figure S1).

\section{Extracellular vesicle characterization Transmission electron microscopy (TEM)}

Ten microliters of EV suspension (stored at $4{ }^{\circ} \mathrm{C}$ for $12 \mathrm{~h}$ after purification) were placed on Formvar-covered copper grids (Agar Scientific, Stansted, UK) and stained with $1 \%$ phosphotungstic acid (Electron Microscopy Sciences, Hatfield, PA) for $30 \mathrm{~s}$. The preparations were observed in a JEM-1010 microscope (Jeol, Peabody, MA). For cryogenic electron microscopy (Cryo-TEM), $10 \mu \mathrm{l}$ of EV suspension were directly observed in a JEM-2100 LaB6 microscope (Jeol). The Digital Micrograph 2.0x software (Gatan, Pleasanton, CA) was used.

\section{Nanoparticle tracking analysis}

The EV size distribution was determined in a Nanosight NS300 (Malvern Panalytical, Malvern, UK) with the $488 \mathrm{~nm}$ laser and the NTA 3.2.16 software (Malvern). The average of the results obtained from four one-minute reads is reported.

\section{Flow cytometry}

One milliliter of EV suspension was stained with $1 \mu \mathrm{l}$ of rabbit anti-mouse Rab7 antibody (clone ab137029, Abcam, San Francisco, CA) at $4{ }^{\circ} \mathrm{C}$ overnight. The EVs were washed by ultracentrifugation at $160,000 \mathrm{xg}$ for 90 min in an Optima MAX ultracentrifuge (Beckman Coulter) with a TLS55 rotor; $200 \mu \mathrm{l}$ of suspension were collected from the bottom of the tube and stained with $1 \mu \mathrm{l}$ of FITC-labeled anti-rabbit IgG antibody (clone 31,635, Thermo Fisher Scientific, Waltham, MA) at room temperature for $1 \mathrm{~h} .800 \mu \mathrm{l}$ of Hanks' balanced salt solution (Gibco) were added and the EVs were washed again. $200 \mu \mathrm{l}$ of suspension were collected from the bottom of the tube. Ten min before analysis, $1 \mu \mathrm{l}$ of APC-labeled annexin V (BioLegend, San Diego, CA) and $200 \mu \mathrm{l}$ of annexin $\mathrm{V}$ binding buffer (BioLegend) were added (annexin $\mathrm{V}$ is a protein that binds to phosphatidylserine when this phospholipid is present in the outer membrane layer). The EVs were analyzed in a FACSCalibur (Beckton Dickinson, San Jose, CA) flow cytometer previously washed with $1 \mathrm{~L}$ of $0.45 \mu \mathrm{m}$-filtered $0.5 \%$ sodium hypochlorite and $5 \mathrm{~L}$ of $0.45 \mu \mathrm{m}$-filtered distilled water. At least 900,000 events were acquired for each sample. Data were analyzed with FlowJo 10.4.2 software (FlowJo LLC, Ashland, OR).

\section{Extracellular vesicle chemical}

\section{composition}

\section{Protein analysis}

Proteins were extracted from EV suspensions with $15 \%$ (final concentration) of trichloroacetic acid, and analyzed by SDS-polyacrylamide gel electrophoresis (PAGE; $2 \mu \mathrm{g}$ of protein per well) with $5 / 12 \%$ polyacrylamide gels and run with Tris/glycine/SDS (25 mM/192 mM/1\%) buffer. The electrophoresis was performed at $30 \mathrm{~mA}$ for $90 \mathrm{~min}$; the proteins were visualized with silver staining (GE Healthcare) and analyzed with ImageJ software $1.50 \mathrm{i}$ (National Institutes of Health, Bethesda, MD). Alternatively, for the Western blot analysis, the proteins were transferred to a $0.45 \mu \mathrm{m}$ pore nitrocellulose membrane (Amersham Biosciences, Little Chalfont, UK) using an electrotransfer tank $\left(0.8 \mathrm{~mA} / \mathrm{cm}^{2}\right.$ for $\left.90 \mathrm{~min}\right)$. The membrane was blocked with $5 \%$ skimmed milk in PBS for $1 \mathrm{~h}$ at $4^{\circ} \mathrm{C}$. The membrane was then incubated with 1:7,000 rabbit anti-M. tuberculosis H37Rv soluble extract (MTSE) serum [which was prepared by immunizing a rabbit with $1 \mathrm{mg}$ of MTSE mixed 1:1 with incomplete 
Freund's adjuvant (Sigma Aldrich, St. Louis, MO); a total of two immunizations were performed, on day 0 and day 28 , and blood was obtained on day 42]. The membrane was left at $4{ }^{\circ} \mathrm{C}$ overnight. After extensive washing with PBS, peroxidase-labeled anti-rabbit IgG antibody (clone Poly4064, BioLegend), diluted to 1:2,000 was added, and the bands were visualized with 3,3'-diaminobenzidine (Sigma-Aldrich) and $\mathrm{H}_{2} \mathrm{O}_{2}$.

\section{Lipid analysis}

Lipids were extracted from EV suspensions with the Folch method, ${ }^{21}$ adding an equal volume of chloroform/methanol 2:1. After chloroform/methanol evaporation, lipids were analyzed by thin-layer chromatography using Silica gel $60 \mathrm{~F}_{254}$ plates (Merck, Darmstadt, Germany) and chloroform/methanol/acetic acid/water 65:25:8:4 as the mobile phase. After drying for $2 \mathrm{~h}$, the plates were treated with $1 \%$ ninhydrin in ethanol (which reacts to lipids with free amino groups, such as phosphatidylserine and phosphatidylethanolamine $)^{22}$ or with $5 \%$ sulphuric acid in ethanol (which reveals the total carbon content of each sample). ${ }^{23}$ The plates were photographed and analyzed with ImageJ $1.50 \mathrm{i}$ software. Lipids from $1 \times 10^{6}$ J774A.1 cells were extracted and analyzed at the same time as the EV lipids, in order to determine the position of phosphatidylserine and phosphatidylethanolamine on the plates (phosphatidylethanolamine migrates faster than phosphatidylserine). ${ }^{24}$

\section{In vitro model of mycobacterium tuberculosis infection}

J774A.1 cells were cultured to near $100 \%$ confluence in 24-well plates (Corning) and infected with M. tuberculosis $\mathrm{H} 37 \mathrm{Rv}$ at $\mathrm{MOI}=10$ for $3 \mathrm{~h}$. Cells were washed, and extracellular mycobacteria were eliminated with 10 $\mu \mathrm{g} / \mathrm{ml}$ amikacin (Bruluart, Tultitlán, Mexico). After $1 \mathrm{~h}$, the cells were washed and incubated in DMEM with $7 \%$ of EV-reduced fetal calf serum, $200 \mu \mathrm{l}$ of annexin V binding buffer (BioLegend) and $2.5 \mu \mathrm{g}$ (the protein concentration was determined by absorbance at 230nm) of EVs or annexin V-blocked EVs. [These EVs were incubated with $1 \mu \mathrm{l}$ of unlabeled annexin $\mathrm{V}$ (BioLegend) for $15 \mathrm{~min}$ before use; the blocking of phosphatidylserine was confirmed by adding $1 \mu \mathrm{l}$ of APC-labeled annexin V (BioLegend) and analyzing by flow cytometry]. After $44 \mathrm{~h}$, the culture supernatants were collected and stored at $-70^{\circ} \mathrm{C}$, and $\mathrm{CFU}$ were determined by lysing the cells with $500 \mu 1$ of $0.1 \%$ Triton X-100 (Sigma-Aldrich) for $7 \mathrm{~min}$ and performing serial dilutions in $7 \mathrm{H} 9$ broth; $10 \mu \mathrm{l}$ of each dilution were plated on Middlebrook 7H10 agar with OADC growth supplement. Colonies were counted at days 7 and 15 . The culture supernatants were analyzed with a CBA Mouse Inflammation Kit (BD Biosciences, San Jose, CA) to determine the concentrations of IL-6, IL10, IL-12p70, MCP-1 and TNF- $\alpha$; the samples were acquired in a FACSAria III flow cytometer (BD Biosciences). Data were analyzed with FlowJo 10.4.2 software.

\section{Mouse model of pulmonary tuberculosis}

BALB/c mice ( 8 weeks of age, male) were anesthetized and inoculated intra-tracheally with $2.5 \times 10^{5} \mathrm{CFU}$ of $M$. tuberculosis H37Rv in $100 \mu$ l of endotoxin-free sterile PBS. ${ }^{25}$ Thirty days after infection, each mouse (10 mice per group) received intra-peritoneal injections of $100 \mu \mathrm{l}$ of $0.85 \%$ $\mathrm{NaCl}$ alone or with $2.5 \mu \mathrm{g}$ of vesicle protein of S-EV or Mtb-EV, three times per week (as previously reported for other anti-tuberculosis treatments tested in this mouse model). ${ }^{26,27}$ Mice were euthanized after 30 or 60 days of treatment. The right lung of each mouse was homogenized in a Fast-prep 24 (MP Biomedicals, Santa Ana, CA) with $1 \mathrm{ml}$ of sterile $0.05 \%$ Tween 80 in PBS, serial dilutions in PBSTween $0.05 \%$ broth were performed, and $10 \mu \mathrm{l}$ of each dilution were plated on Middlebrook $7 \mathrm{H} 10$ agar with OADC growth supplement; colonies were counted after 14 and 21 days. The left lung of each mouse was perfused with absolute ethanol for at least $24 \mathrm{~h}$ and embedded in paraffin. Four micrometer-thick slides were obtained and stained with hematoxylin and eosin, and the pneumonic area in each slide was determined with the image analysis software Leica Application suite 4.2 (Leica Microsystems, Wetzlar, Germany). For this analysis, images from different fields of the slide (observed at 25x magnification) were stitched together to create an image of the whole lung section. The pneumonic area (the parenchyma that had undergone hepatization) was marked off manually. To determine the total parenchymal area, the perimeter of the lung was marked off manually, and the areas corresponding to the blood vessels and to the respiratory tree were subtracted from this total lung area. The percentage of the pneumonic area, compared to the total parenchymal area, was calculated for each case.

\section{Statistical analysis}

Data were analyzed with the SigmaPlot 12.2.0.45 software (Systat Software, San Jose, CA). Wilcoxon signed-rank test or Mann-Whitney U test were used to compare two groups, while Kruskal-Wallis test followed by Tukey's 
test, or one-way analysis of variance (ANOVA) followed by Tukey's test, were used to compare more than two groups. The Kaplan-Meier estimator was used for survival analysis. A $p$-value $<0.05$ was considered significant.

\section{Results}

The EVs released by infected J774A.I macrophages differ in size and chemical composition from spontaneouslyreleased EVs

J774A.1 cells spontaneously release EVs to the culture supernatant. To determine if infection with $M$. tuberculosis modifies the size and the chemical composition of these EVs, we purified the EVs released spontaneously from J774A.1 cells (S-EV) and the EVs released from J774A.1 cells infected with M. tuberculosis H37Rv for $4 \mathrm{~h}$ (Mtb-EV). Figure 1A shows the absorbance at $230 \mathrm{~nm}$ of each fraction obtained from the size-exclusion chromatography performed on the ultra-centrifuged culture supernatant of $M$. tuberculosisinfected J774A.1 cells. Fraction 26 corresponded to the first fraction with peak absorbance that contained EVs (Figure 1C); earlier fractions, such as fraction 15 , contained no EVs (Figure 1B), and later fractions were composed mainly of culture media. For all subsequent experiments, we used the EV-containing fractions (Figure $1 \mathrm{C}$ is a representative image). Cryo-TEM analysis confirmed that the purified EVs conserved their integrity and had bilayered membranes (Figure 1D), and showed that S-EV (Figure 1E) were smaller than Mtb-EV (Figure 1F). Nanoparticle tracking analysis confirmed that most S-EV had a size of around $130 \mathrm{~nm}$, while most Mtb-EV had a size of around $150 \mathrm{~nm}$ (Figure 1G).

SDS-PAGE indicated that S-EV and Mtb-EV had a similar protein composition. However, a $22 \mathrm{kDa}$ protein is only found in Mtb-EV (Figure 2A and B, arrows). Western blot analysis of Mtb-EV showed that they contained $M$. tuberculosis H37Rv antigens of 30, 40, 50 and $100 \mathrm{kDa}$, which were not present in S-EV. These antigens are specific of M. tuberculosis, since they were not detected in EVs released from J774A.1 cells infected for $4 \mathrm{~h}$ with $M$. fortuitum (Mf-EV), a mycobacteria with many cell wall components similar to those of M. tuberculosis ${ }^{28,29}$ (Figure 2C). Mtb-EV tended to have increased phosphatidylserine in their outer membrane layer and an increased percentage of phosphatidylserine-containing EVs in comparison with S-EV (Figure 2D and E). Accordingly, Mtb-EV had a significantly higher content of phosphatidylserine, as assessed by thin-layer chromatography, than S-EV (Figure 2F), while these two EVs did not differ in their total carbon content (Figure 2G).

\section{The EVs released by J774A.I} macrophages reduce the bacterial load in both in vitro and in vivo models of mycobacterium tuberculosis infection

The expression of phosphatidylserine on the outer layer of cell membranes has anti-inflammatory effects on immune cells. ${ }^{30,31}$ To determine if the phosphatidylserine found on S-EV and Mtb-EV affected the bactericidal capacity or the cytokine production of mouse macrophages, J774A.1 cells were infected with $M$. tuberculosis $\mathrm{H} 37 \mathrm{Rv}$ and then treated with EVs or annexin V-blocked EVs. Figure 3A shows that unlabeled annexin $\mathrm{V}$ prevented the binding of APClabeled annexin $\mathrm{V}$ to Mtb-EV, confirming that unlabeled annexin $\mathrm{V}$ masks the phosphatidylserine on the outer layer of the EV membranes. S-EV reduced the bacterial load in M. tuberculosis-infected J774A.1 cells, and this effect was not observed when the phosphatidylserine on S-EV was blocked with annexin V (Figure 3B). S-EV also reduced the production of MCP-1 and TNF- $\alpha$ by M. tuberculosisinfected J774A.1 cells, and this effect was reversed when the phosphatidylserine on S-EV was blocked with annexin $\mathrm{V}$ (Figure 3C). No differences were observed in IL-6 production (Figure $3 \mathrm{C}$ ), and no IFN- $\gamma$, IL-12 p70 or IL10 production was detected at this time (data not shown). Mtb-EV did not change the bacterial load or the cytokine production in $M$. tuberculosis-infected J774A.1 cells (Figure 3B and C).

To determine if the reduction in bacterial load also occurred in vivo, we used a mouse model of pulmonary tuberculosis, in which mice are infected intra-tracheally with M. tuberculosis H37Rv. Infected mice were treated intra-peritoneally with saline solution, with S-EV or with Mtb-EV three times per week. EVs had no significant effect on mice survival (Figure 4A), but both S-EV and Mtb-EV decreased the lung bacterial load after 60 days of treatment (Figure 4B). However, treatment with S-EV or with Mtb-EV had no effect on the lung pneumonic area in M. tuberculosis-infected mice (Figure 4C and D).

\section{Discussion}

EVs are released by most cell types and, since they transport lipids, proteins and nucleic acids from the donor cell to the target cell, they constitute an efficient cellular 
A

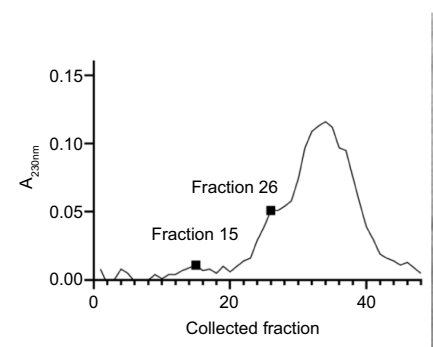

Collected fraction
B

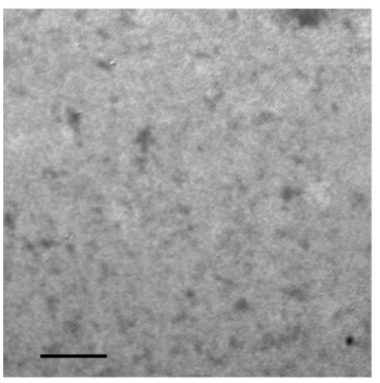

C

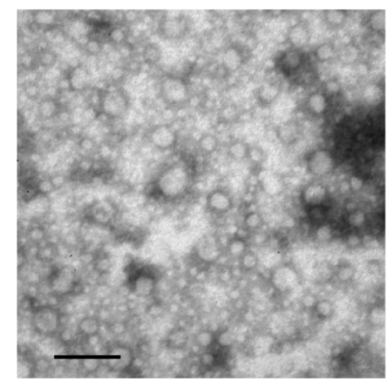

D

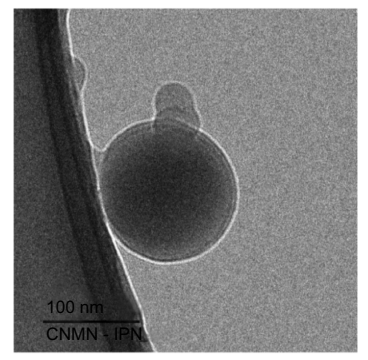

E

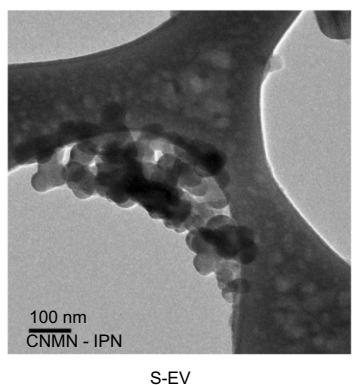

F

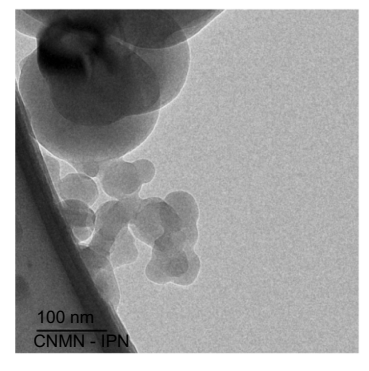

G

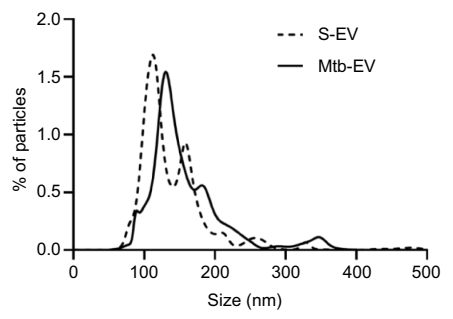

Figure I EVs released by infected J774A.I macrophages are larger than spontaneously-released EVs. EVs were purified from J774A.I cells that had been infected with M. tuberculosis H37Rv for $4 \mathrm{~h}$ (Mtb-EV). (A) Absorbance at $230 \mathrm{~nm}$ of the fractions obtained from the size-exclusion chromatography performed on the ultra-centrifuged culture supernatant of $M$. tuberculosis-infected J774A.I cells. (B) TEM analysis of fraction I5. (C) TEM analysis of fraction 26. The results are representative of four experiments. (D) Cryo-TEM analysis of a fraction containing Mtb-EV. (E) Cryo-TEM analysis of EVs released spontaneously from J774A.I cells (S-EV). (F). Cryo-TEM analysis of Mtb-EV G. Nanoparticle tracking analysis of S-EV and Mtb-EV, showing the percentage of particles in a sample that are of the indicated size. The result is representative of four experiments. In TEM and Cryo-TEM, bars $=100 \mathrm{~nm}$.

Abbreviations: M. tuberculosis, Mycobacerium tuberculosis; TEM, transmission electron microscopy; EVs, extracellular vesicles.

communication mechanism. Since the composition of EVs can change in response to the activation signal that leads to its production, ${ }^{11}$ in this work we compared the EVs released spontaneously from J774A.1 macrophages (SEV) with those released from J774A.1 macrophages that had been infected with M. tuberculosis H37Rv for $4 \mathrm{~h}$ (Mtb-EV). S-EV were smaller than Mtb-EV, and their protein composition, as assessed by SDS-PAGE, was broadly similar, differing only in a $22 \mathrm{kDa}$ protein that was present in Mtb-EV but not in S-EV. As expected, MtbEV contained mycobacterial antigens; the most abundant of these antigens had molecular weights of 30, 40, 50 and $100 \mathrm{kDa}$, but not of $22 \mathrm{kDa}$. These antigens were specific of M. tuberculosis, since they were not detected in EVs released from J774A.1 macrophages that had been infected with M. fortuitum for $4 \mathrm{~h}$ (Mf-EV). M. fortuitum has cell wall components that cross-react with the cell wall components of M. tuberculosis, ${ }^{28,29}$ but these antigens, or any other antigen shared between these two mycobacteria, were not present in the Mf-EV, since these EVs did not react with an anti-M. tuberculosis $\mathrm{H} 37 \mathrm{Rv}$ soluble extract (MTSE) serum.

Rab7 is a characteristic marker of exosomes, because these EVs derive from late endosomes. Rab7 is present in EVs released from mouse macrophages and from human neutrophils infected with M. tuberculosis. ${ }^{32,33}$ Phosphatidylserine is found in the outer membrane layer of exosomes, ${ }^{34}$ ectosomes, ${ }^{35}$ microvesicles ${ }^{36}$ and even synaptic vesicles. ${ }^{37}$ It is a phospholipid with anti-inflammatory effects, and its transport from the inner to the outer membrane layer is a characteristic feature of cellular apoptosis. ${ }^{30}$ However, the enrichment of phosphatidylserine in EVs is not associated with apoptosis of the EVproducing cell. ${ }^{38}$ We confirmed that S-EV and Mtb-EV contained Rab7 and, as expected, they also contained phosphatidylserine in their outer membrane layer. ${ }^{33} \mathrm{Mtb}$ EV had an increased content of phosphatidylserine, as assessed by thin-layer chromatography (a method with 
A

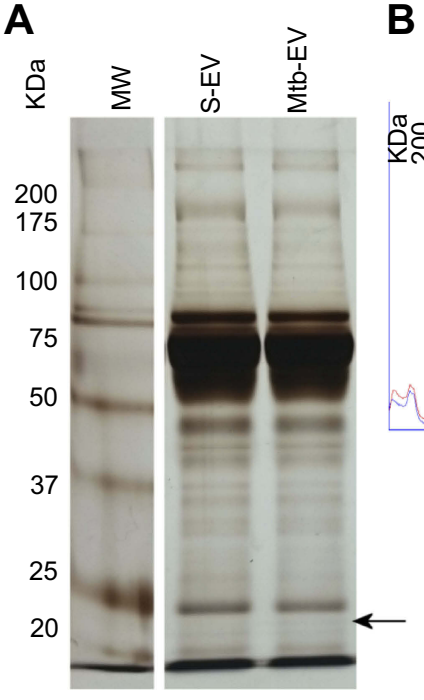

D S-EV

갈

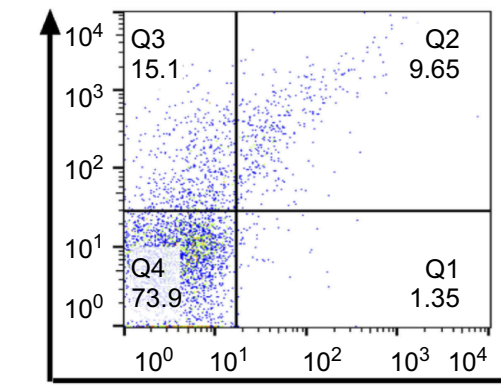

B

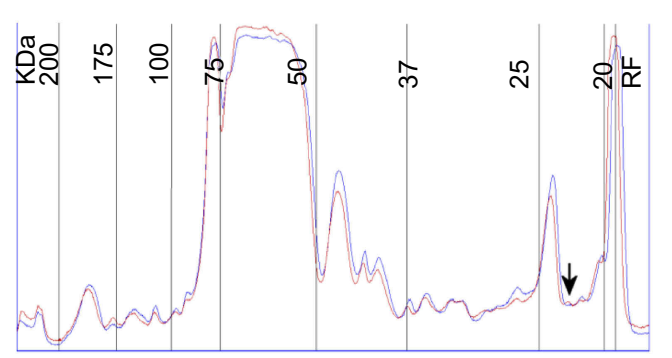

MW

S-EV

Mtb-EV
C

$\stackrel{\widetilde{Q}}{\underline{\mathrm{C}}}$

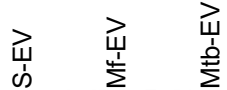

150

100

75

50

37

25

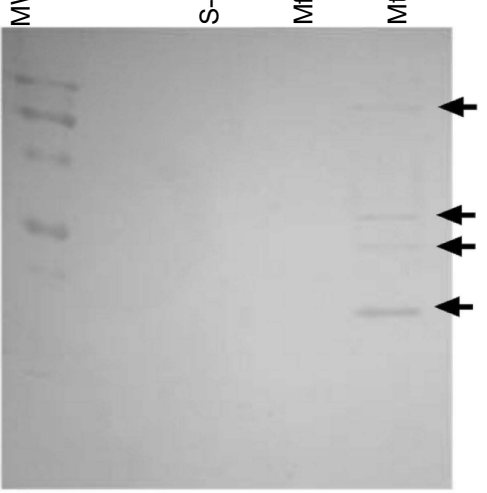

\section{F}

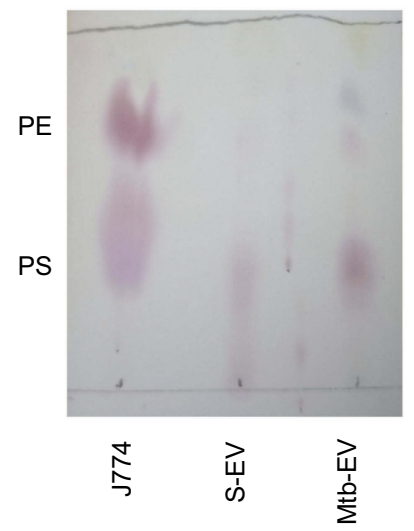

Rab 7
Mtb-EV

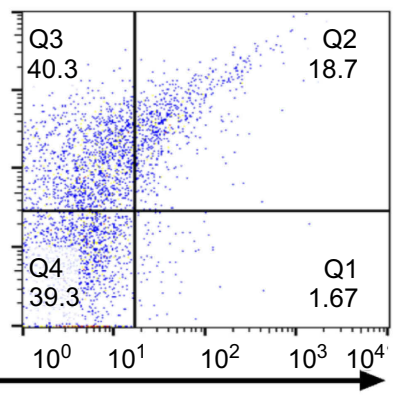

G

\section{E}
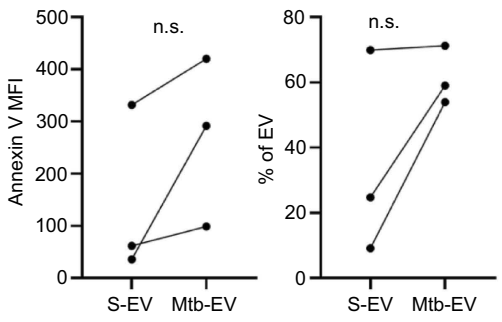

Total carbon
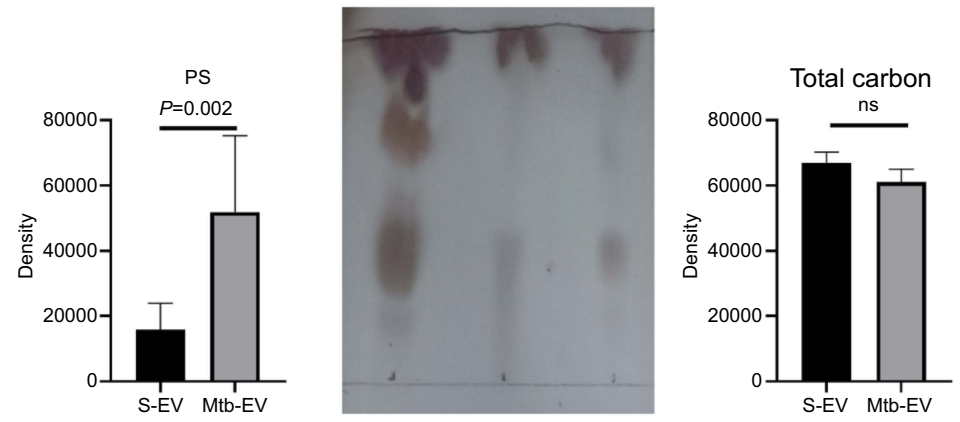

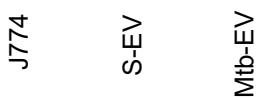

Figure 2 EVs released by infected J774A.I macrophages contain Mycobacterium tuberculosis antigens and are enriched in phosphatidylserine. EVs were purified from J774A.I cells (S-EV), from J774A.I cells that had been infected with M. fortuitum for $4 \mathrm{~h}$ (Mf-EV) or from J774A.I cells that had been infected with M. tuberculosis H37Rv for $4 \mathrm{~h}$ (Mtb-EV). (A) S-EV and Mtb-EV were analyzed by SDS-polyacrylamide gel electrophoresis (SDS-PAGE), the arrow indicates a $22 \mathrm{kDa}$ protein exclusive to Mtb-EV. MW, molecular weight marker. (B) Densitometric analysis of the SDS-PAGE in A, the arrow indicates a $22 \mathrm{kDa}$ protein exclusive to Mtb-EV. MW, molecular weight marker. RF, Running front. (C) S-EV, Mf-EV and Mtb-EV were analyzed by Western blot, with an anti-M. tuberculosis H37Rv soluble extract (MTSE) serum as primary antibody. The arrows indicate 30, 40, 50 and I00 kDa antigens. MW, molecular weight marker. (D) S-EV and Mtb-EV were stained with rabbit anti-mouse Rab7 antibody and FITC-labeled anti-rabbit lgG antibody, and with APC-labeled annexin V [which binds to phosphatidylserine (PS)] and were analyzed by flow cytometry. (E) The APC-labeled annexin V mean fluorescence intensity (MFI) and the percentage of phosphatidylserine-containing EVs from three experiments are shown. Wilcoxon signed-rank test. n.s, not significant. (F, G) Lipids were extracted from J774A.I cells, from S-EV and from Mtb-EV, and analyzed by thin-layer chromatography, with ninhydrin [which reacts with lipids with free amino groups, such as phosphatidylserine (PS) and phosphatidylethanolamine (PE)] or with sulphuric acid (which reveals the total carbon content of each sample). One plate representative of three is shown. The spot density is expressed as area under the curve. Graphs represent mean and SEM of three independent experiments. Mann-Whitney $U$ test.

Abbreviations: M. tuberculosis, Mycobacerium tuberculosis; M. fortuitum, Mycobacterium fortuitum; TEM, transmission electron microscopy; EVs, extracellular vesicles; SD, standard deviation; n.s., not significant. 

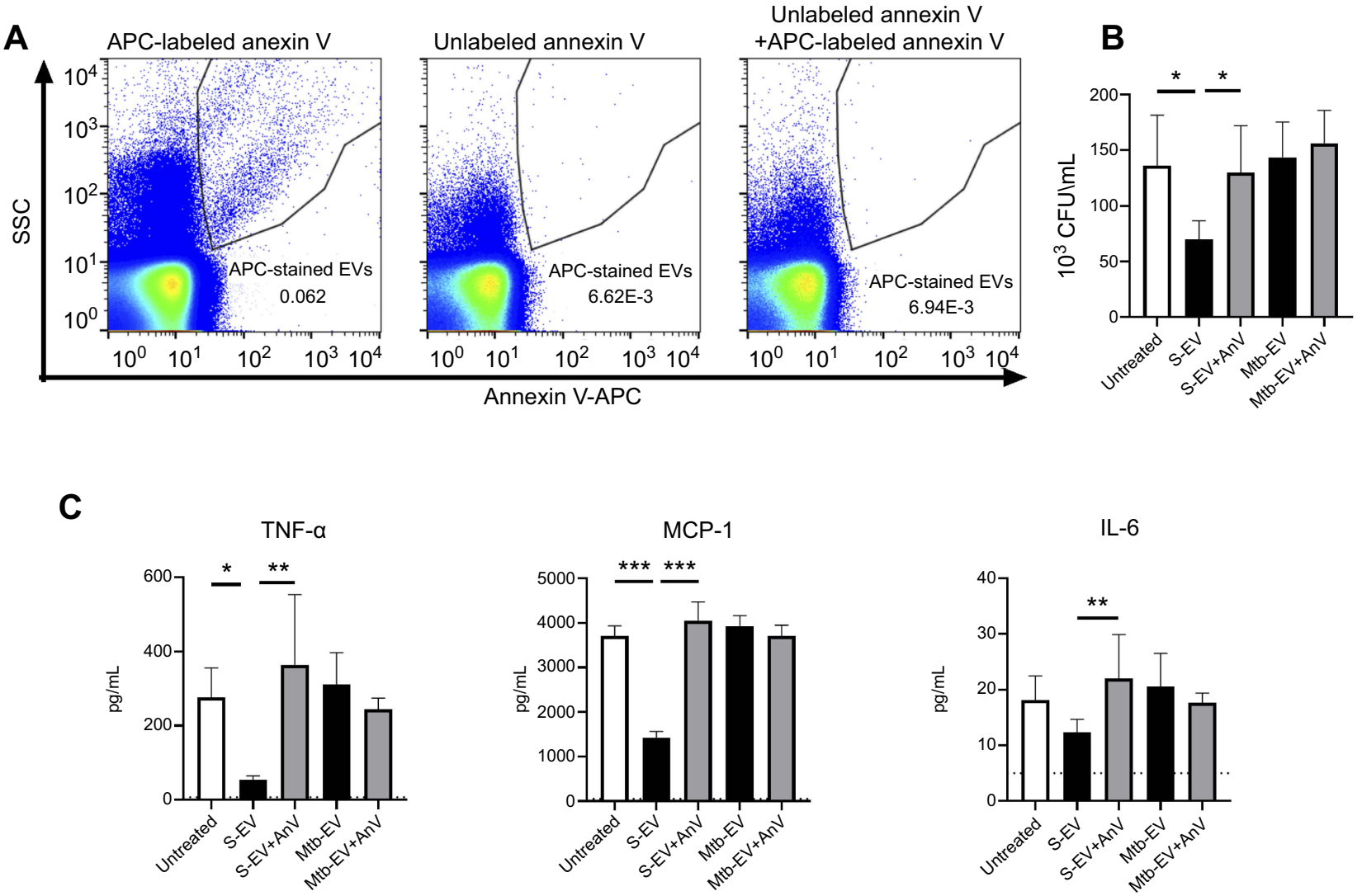

Figure $3 \mathrm{EVs}$ released by J774A.I macrophages reduce the bacterial load and the production of MCP-I and TNF- $\alpha$ through a phosphatidylserine-dependent mechanism. J774A.I cells were infected with M. tuberculosis $\mathrm{H} 37 \mathrm{Rv}$ at a $\mathrm{MOI}=10$ for $3 \mathrm{~h}$; extracellular mycobacteria were eliminated with $10 \mu \mathrm{g} / \mathrm{ml}$ amikacin for I h and $2.5 \mu \mathrm{g}$ of EVs or annexin $\mathrm{V}(\mathrm{AnV})$-blocked EVs were added. After $44 \mathrm{~h}$, bacterial load and cytokine concentrations were determined. (A) Left panel: Mtb-EV were stained with APC-labeled annexin $\vee$ and analyzed by flow cytometry. Middle panel: Mtb-EV were incubated with unlabeled annexin $V$ and analyzed by flow cytometry. Right panel: Mtb-EV were incubated with unlabeled annexin $V$ for 15 min and then stained with APC-labeled annexin $V$ and analyzed by flow cytometry. (B) CFU in infected J774A.I cells, incubated with medium alone (untreated), with EVs or with annexin V-blocked EVs. (C) Concentrations of TNF- $\alpha$, MCP-I and IL-6 in the supernatants of infected J774A.I cells, incubated with medium alone (untreated), with EVs or with annexin V-blocked EVs (Mtb-EV-AnV). Graphs represent mean and SEM of three independent experiments. Dotted lines indicate the limit of detection of the assay. One-way ANOVA with Tukey's test: *, $p<0.05 ; * * p<0.0 \mathrm{I} ; * * * p<0.00 \mathrm{I}$. S-EV, EVs purified from J774A.I cells; Mtb-EV, EVs purified from J774A.I cells that had been infected with $M$. tuberculosis H37Rv for $4 \mathrm{~h}$.

Abbreviations: M. tuberculosis, Mycobacerium tuberculosis; EVs, extracellular vesicles; SD, standard deviation.

higher sensitivity than flow cytometry). This increase in phosphatidylserine content could be caused by the activity of phospholipid scramblase 1(PLSCR1), since this enzyme transports phosphatidylserine from the inner to the outer layer of cellular membranes ${ }^{39}$ and is overexpressed in patients with tuberculosis. ${ }^{40}$

Liposomes with phosphatidylserine in their outer membrane layer and phosphatidic acid in their inner membrane layer reduce the bacterial load in in vitro and in vivo models of tuberculosis, ${ }^{41}$ and the phagocytosis of apoptotic bodies inhibits the production of TNF- $\alpha$ and induces the release of TGF- $\beta$ by macrophages., ${ }^{31,42}$ The phosphatidylserine receptor stabilin-2 is necessary for these processes. ${ }^{42}$ Ectosomes derived from human neutrophils express phosphatidylserine in their outer membrane layer, and these ectosomes decrease the production of TNF- $\alpha$ and induce the release of TGF- $\beta$ by human macrophages. ${ }^{43}$ To determine if S-EV and Mtb-EV had an effect on the bactericidal capacity or the cytokine production of mouse macrophages, J774A.1 cells were infected with $M$. tuberculosis $\mathrm{H} 37 \mathrm{Rv}$ and then treated with $\mathrm{EV}$ s or with annexin V-blocked EVs. S-EV reduced the bacterial load and the production of MCP-1 and TNF- $\alpha$ in M. tuberculosis-infected J774A.1 cells, and these effects were reversed when phosphatidylserine was blocked with annexin $\mathrm{V}$, indicating that the anti-mycobacterial and anti-inflammatory effects of S-EV are phosphatidylserine-dependent. In a previous study, we reported that EVs released by $M$. tuberculosis-infected human neutrophils induce autophagy and reduce the bacterial 
A

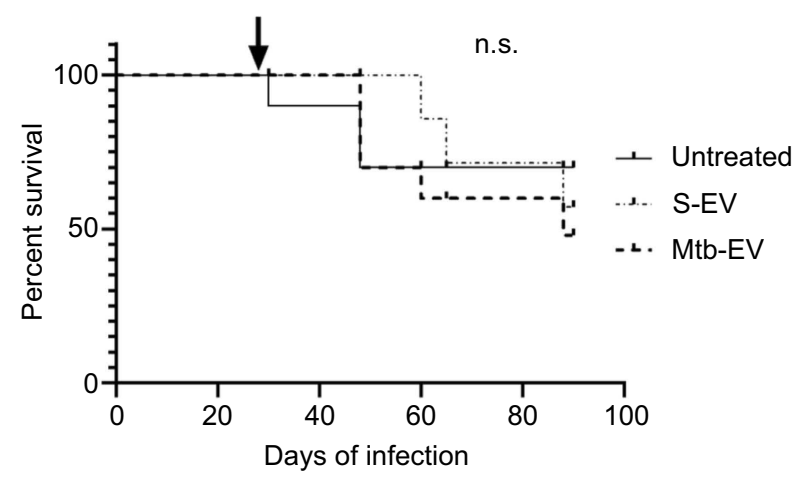

B

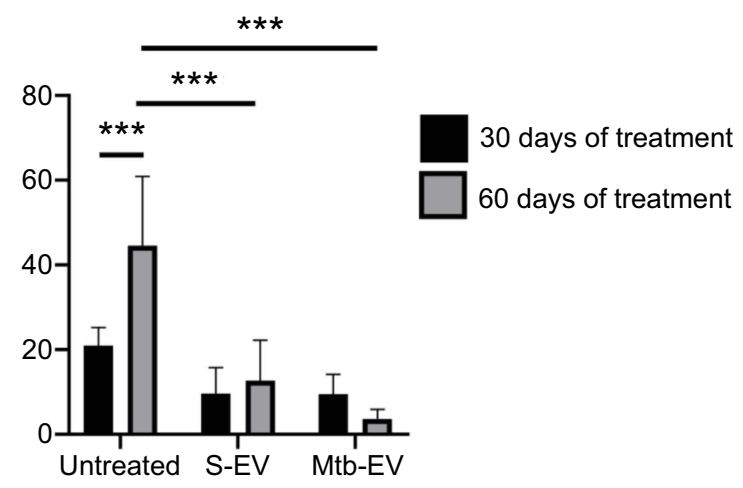

C

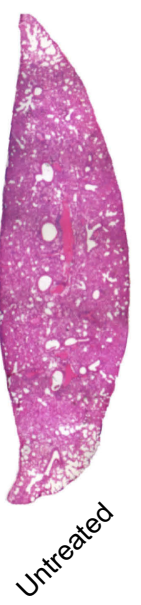

30 days of treatment
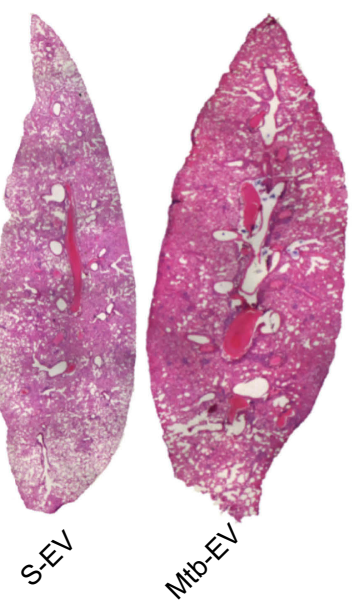

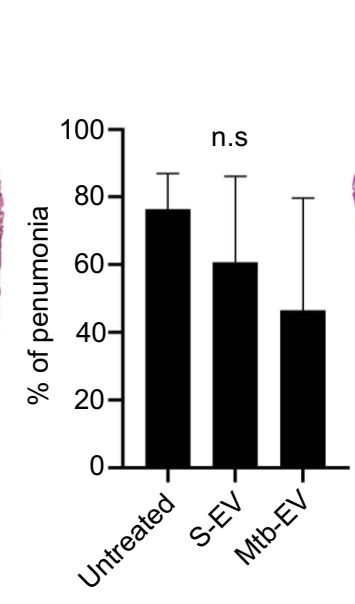

D

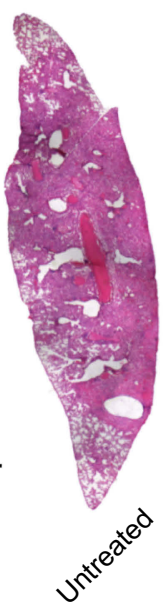

60 days of treatment

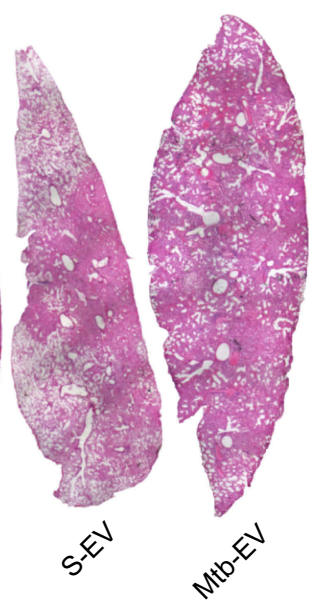

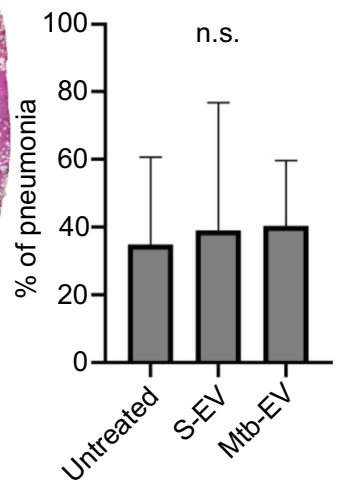

Figure $4 \mathrm{EVs}$ released by J774A.I macrophages reduce the bacterial load in a mouse model of pulmonary tuberculosis. BALB/c mice were inoculated intra-tracheally with $2.5 \times 10^{5} \mathrm{CFU}$ of $M$. tuberculosis H37Rv. Thirty days after infection, each mouse (10 mice per group) received intra-peritoneal injections (three times per week) of saline solution, alone, with EVs purified from J774A.I cells (S-EV) or with EVs purified from J774A.I cells that had been infected with M. tuberculosis H37Rv for $4 \mathrm{~h}$ (Mtb-EV). Mice lungs were analyzed after 30 or 60 days of treatment. (A) Mice survival. The arrow indicates the beginning of the indicated treatments. (B) Lung bacterial load. The graph represents mean and SEM of one experiment (representative of three independent experiments). Two-way ANOVA with Tukey's test: **** $p<0.00 \mathrm{I}$. (C) Lung sections and lung pneumonic areas at 30 days of treatment. The graph represents mean and SEM of one experiment (representative of three independent experiments). Kruskal-Wallis test: n.s., not significant. (D) Lung sections and lung pneumonic are at 60 days of treatment. The graph represents mean and SEM of one experiment (representative of three independent experiments). Kruskal-Wallis test.

Abbreviations: M. tuberculosis, Mycobacerium tuberculosis; CFU, colony forming unit; EVs, extracellular vesicles; SD, standard deviation; n.s., not significant.

load in infected macrophages. ${ }^{19}$ It remains to be determined if $\mathrm{S}-\mathrm{EV}$ also reduce the bacterial load by inducing autophagy in macrophages. In contrast to S-EV, Mtb-EV had no effect on the bacterial load or on cytokine production in M. tuberculosisinfected J774A.1 cells, and the blocking of phosphatidylserine with annexin $\mathrm{V}$ had no detectable effect on the activity of these EVs. It has been shown that EVs from macrophages ${ }^{12}$ or neutrophils ${ }^{19}$ infected with $M$. tuberculosis contain TLR ligands, so it is possible that Mtb-EV-induced TLR activation might cancel the anti-inflammatory effects of the phosphatidylserine from these EVs.
As a first approach to evaluate the therapeutic potential of EVs, we tested the S-EV and Mtb-EV in a mouse model of pulmonary tuberculosis. In a previous study, membrane vesicles released by $M$. tuberculosis were administered subcutaneously to mice ( $2.5 \mu \mathrm{g}$ of vesicle protein per mouse). These vesicles elicited a protective immune response against an aerosol infection with $M$. tuberculosis that was comparable to the response elicited by immunization with Bacillus Calmette-Guérin (BCG). ${ }^{44}$ However, when these same membrane vesicles were administered intra-tracheally to mice $(25 \mu \mathrm{g}$ of vesicle protein per mouse), they increased 
the bacterial load and the lung pathology after an aerosol infection with M. tuberculosis. ${ }^{45}$ In our model, which represents the treatment of an ongoing tuberculosis infection rather than a vaccine strategy, we administered $2.5 \mu \mathrm{g}$ of vesicle protein per mouse by intra-peritoneal injection, to achieve faster distribution of the EVs. ${ }^{46}$ We observed that $\mathrm{S}-\mathrm{EV}$ and Mtb-EV had no effect on the survival or the lung pneumonic area of mice infected intra-tracheally with $M$. tuberculosis $\mathrm{H} 37 \mathrm{Rv}$, but both EVs significantly reduced the lung bacterial load after 60 days of treatment.

Our results indicate that $\mathrm{Mtb}-\mathrm{EV}$ decreased the bacterial load in vivo but not in vitro, which suggests that other EV components, in addition to phosphatidylserine (which is increased in Mtb-EV, compared to S-EV), also elicit an anti-mycobacterial effect. EVs released by $\mathrm{J774}$ macrophages infected with M. tuberculosis H37Rv contain mycobacterial antigens, including the antigen 85 (Ag85) complex (Rv3804c, Rv1886c and Rv0129c) and ESAT-6 (Rv3875). ${ }^{12}$ These antigens are actively sorted into the exosomes by Rab27, since the exosomes released from Rab27-deficient macrophages contain fewer mycobacterial antigens, and serum exosomes from Rab27-deficient mice induce lower production of TNF- $\alpha$ by bone marrow-derived macrophages than serum exosomes from wild-type mice. ${ }^{16}$ It has been shown that the presence of mycobacterial antigens in EVs released by infected neutrophils can lead to mycobacterial clearance in vitro, ${ }^{19}$ and antigen-containing exosomes contribute to the $\mathrm{T}$ cell anti-mycobacterial response in a mouse model of $M$. tuberculosis infection. ${ }^{16,32}$ T-cell activation by Mtb-EV, which would occur in vivo but not in vitro, could explain why Mtb-EV decreased the bacterial load in the mouse model of pulmonary tuberculosis but not in $M$. tuberculosis-infected J774A.1 cells.

In future studies, it will be necessary to evaluate the efficiency of S-EV and Mtb-EV in combination with the conventional anti-tuberculosis pharmacologic treatment to determine if these EVs can shorten the duration of the treatment. It will also be interesting to evaluate if the administration of drugs that increase the production of EVs, such as statins, ${ }^{47}$ is beneficial during tuberculosis treatment, and if EVs are effective against multi-drugresistant $M$. tuberculosis strains.

In conclusion, we report that EVs released by J774A.1 macrophages reduce the bacterial load in in vitro and in vivo models of $M$. tuberculosis infection. The effects of the EVs released spontaneously by J774A.1 macrophages (S-EV) are partially dependent on the presence of phosphatidylserine in the outer membrane layer of these EVs, but the effects of the EVs released by M. tuberculosisinfected J774A.1 macrophages (Mtb-EV) are independent of this mechanism.

\section{Acknowledgments}

We thank Raúl Borja-Urbi from Centro de Nanociencias, Micro y Nanotecnologías, Instituto Politécnico Nacional, for his assistance in the use of the JEM-2100 microscope. We thank M. Teresa Horn for English language copy-editing. Funding was provided by Consejo Nacional de Ciencia y Tecnología (CONACYT grant 221002to IEG and FC 20151/115 to RHP) and by Secretaría de Investigación y Posgrado (SIP), Instituto Politécnico Nacional (IPN). This work was partially carried out with equipment of "Laboratorio Nacional para Servicios Especializados de Investigación, Desarrollo e Innovación $(\mathrm{I}+\mathrm{D}+\mathrm{i})$ para Farmoquímicos y Biotecnológicos", LANSEIDI-FarBiotec-CONACyT, which is part of Unidad de Desarrollo e Investigación en Bioprocesos (UDIBI)-IPN. MGM, LVF, and VDAJ were recipients of CONACYT fellowships. MIH, LEST, SEP, RCS, JSL and IEG are fellows of the Comisión de Operación y Fomento de Actividades Académicas-IPN. MIH, LEST, SEP, RCS, JSL, IWB and IEG are fellows of Estímulo al Desempeño de los Investigadores-IPN.

\section{Disclosure}

The authors report no conflicts of interest in this work.

\section{References}

1. World Health Organization. Global Tuberculosis Report 2018. Geneva: World Health Organization; 2018.

2. Yee D, Valiquette C, Pelletier M, Parisien I, Rocher I, Menzies D. Incidence of serious side effects from first-line antituberculosis drugs among patients treated for active tuberculosis. Am J Respir Crit Care Med. 2003;167(11):1472-1477. doi:10.1164/rccm.200206-626OC

3. Vasconcellos SEG, Huard RC, Niemann S, et al. Distinct genotypic profiles of the two major clades of mycobacterium africanum. BMC Infect Dis. 2010;10(1):80. doi:10.1186/1471-2334-10-80

4. Nunes-Alves C, Booty MG, Carpenter SM, Jayaraman P, Rothchild AC, Behar SM. In search of a new paradigm for protective immunity to TB. Nat Rev Microbiol. 2014;12(4):289-299. doi:10.1038/nrmicro3230

5. Keane J, Gershon S, Wise RP, et al. Tuberculosis associated with infliximab, a tumor necrosis factor $\alpha$-neutralizing agent. $N$ Engl $J$ Med. 2001;345(15):1098-1104. doi:10.1056/NEJMoa011110

6. Cavalcanti YVN, Brelaz MCA, Neves JKDAL, Ferraz JC, Pereira VRA. Role of TNF-alpha, IFN-gamma, and IL-10 in the development of pulmonary tuberculosis. Pulm Med. 2012;2012:1-10. doi:10.1155/ 2012/745483

7. Meldolesi J. Ectosomes and exosomes-two extracellular vesicles that differ only in some details. Biochem Mol Biol J. 2016;2:3-6. doi:10.21767/2471-8084.100012.

8. Singh PP, Li L, Schorey JS. Exosomal RNA from mycobacterium tuberculosis-infected cells is functional in recipient macrophages. Traffic. 2015;16(6):555-571. doi:10.1111/tra.12278 
9. Garzetti L, Menon R, Finardi A, et al. Activated macrophages release microvesicles containing polarized M1 or M2 mRNAs. J Leukoc Biol. 2014;95(5):817-825. doi:10.1189/jlb.0913485

10. Théry C. Exosomes: secreted vesicles and intercellular communications. F1000 Biol Rep. 2011;3(July):1-8. doi:10.3410/B3-1

11. Li M, Yu D, Williams KJ, Liu M-L. Tobacco smoke induces the generation of procoagulant microvesicles from human monocytes/ macrophages. Arterioscler Thromb Vasc Biol. 2010;30(9):18181824. doi:10.1161/ATVBAHA.110.209577

12. Giri PK, Kruh NA, Dobos KM, Schorey JS. Proteomic analysis identifies highly antigenic proteins in exosomes from M. tuberculosis-infected and culture filtrate protein-treated macrophages. Proteomics. 2010;10(17):3190-3202. doi:10.1002/pmic.200900840

13. Cheng Y, Schorey JS. Exosomes carrying mycobacterial antigens can protect mice against mycobacterium tuberculosis infection. Eur $J$ Immunol. 2013;43(12):3279-3290. doi:10.1002/eji.201343727

14. Singh PP, Smith VL, Karakousis PC, Schorey JS. Exosomes isolated from mycobacteria-infected mice or cultured macrophages can recruit and activate immune cells in vitro and in vivo. J Immunol. 2012;189 (2):777-785. doi:10.4049/jimmunol.1103638

15. Singh PP, LeMaire C, Tan JC, Zeng E, Schorey JS. Exosomes released from m.tuberculosis infected cells can suppress ifn- $\gamma$ mediated activation of naïve macrophages. PLoS One. 2011;6:4. doi:10.1371/journal. pone. 0018564

16. Smith VL, Cheng Y, Bryant BR, Schorey JS. Exosomes function in antigen presentation during an in vivo mycobacterium tuberculosis infection. Sci Rep. 2017;7(March):1-12. doi:10.1038/s41598-016-0028-x

17. Saleh AF, Lazaro-Ibanez E, Forsgard -MA-M, et al. Extracellular vesicles induce minimal hepatotoxicity and immunogenicity. Nanoscale. 2019;11(14):6990-7001. doi:10.1039/c8nr08720b

18. Pullan JE, Confeld MI, Osborn JK, Kim J, Sarkar K, Mallik S. Exosomes as drug carriers for cancer therapy. Mol Pharm. 2019;16 (5):1789-1798. doi:10.1021/acs.molpharmaceut.9b00104

19. Alvarez-Jiménez VD, Leyva-Paredes K, García-Martínez M, et al. Extracellular vesicles released from mycobacterium tuberculosisinfected neutrophils promote macrophage autophagy and decrease intracellular mycobacterial survival. Front Immunol. 2018;9. doi:10.3389/fimmu.2018.00272

20. Glazer AN, Smith EL. Studies on the ultraviolet difference spectra of proteins and polypeptides. J Biol Chem. 1961;236:2942-2947.

21. Folch J, Lees M, Sloane GH. A simple method for the isolation and purification of total lipides from animal tissues. $J$ Biol Chem. 1957;226:497-509.

22. Parker F, Peterson NF. Quantitative analysis of phospholipids and phospholipid fatty acids from silica gel thin-layer chromatograms. $J$ Lipid Res. 1965;6:455-460.

23. Marsh JB, Weinstein B. Simple charring method for determination of lipids. J Lipid Res. 1966;7:574-576.

24. Dyńska-Kukulska K, Ciesielski W. Methods of extraction and thin-layer chromatography determination of phospholipids in biological samples. Rev Anal Chem. 2012;31(1):43-56. doi:10.1515/revac-2011-0030

25. Francisco-Cruz A, Mata-Espinosa D, Ramos-Espinosa O, et al. Efficacy of gene-therapy based on adenovirus encoding granulocyte-macrophage colony-stimulating factor in drug-sensitive and drug-resistant experimental pulmonary tuberculosis. Tuberculosis. 2016;100:5-14. doi:10.1016/j.tube.2016.05.015

26. Rivas-Santiago B, Castañeda-Delgado JE, Rivas Santiago CE, et al. Ability of innate defence regulator peptides IDR-1002, IDR-HH2 and IDR-1018 to protect against mycobacterium tuberculosis infections in animal models. PLoS One. 2013;8(3):e59119-e59119. doi:10.1371/ journal.pone.0059119

27. Hernandez-Pando R, de la Luz Streber M, Orozco H, et al. Emergent immunoregulatory properties of combined glucocorticoid and antiglucocorticoid steroids in a model of tuberculosis. QJM. 1998;91 (11):755-766. doi:10.1093/qjmed/91.11.755
28. Tortola MT, Laneelle MA, Martin-Casabona N. Comparison of two 2,3-diacyl trehalose antigens from mycobacterium tuberculosis and mycobacterium fortuitum for serology in tuberculosis patients. Clin Diagn Lab Immunol. 1996;3(5):563-566.

29. Perley CC, Frahm M, Click EM, et al. The human antibody response to the surface of mycobacterium tuberculosis. PLoS One. 2014;9:6. doi:10.1371/journal.pone.0098938

30. Martin SJ, Reutelingsperger CP, McGahon AJ, et al. Early redistribution of plasma membrane phosphatidylserine is a general feature of apoptosis regardless of the initiating stimulus: inhibition by overexpression of Bcl-2 and Abl. J Exp Med. 1995;182(5):1545-1556. doi:10.1084/jem.182.5.1545

31. Fadok VA, Bratton DL, Konowal A, Freed PW, Westcott JY, Henson PM. Macrophages that have ingested apoptotic cells in vitro inhibit proinflammatory cytokine production through autocrine/paracrine mechanisms involving TGF- $\beta$, PGE2, and PAF. $J$ Clin Invest. 1998;101(4):890-898. doi:10.1172/JCI1112

32. Ramachandra L, Qu Y, Wang Y, et al. Mycobacterium tuberculosis synergizes with ATP to induce release of microvesicles and exosomes containing major histocompatibility complex class II molecules capable of antigen presentation. Infect Immun. 2010;78(12):5116-5125. doi:10.1128/IAI.01089-09

33. González-Cano P, Mondragón-Flores R, Sánchez-Torres LE, et al. Mycobacterium tuberculosis $\mathrm{H} 37 \mathrm{Rv}$ induces ectosome release in human polymorphonuclear neutrophils. Tuberculosis. 2010;90 (2):125-134. doi:10.1016/j.tube.2010.01.002

34. Skotland T, Sandvig K, Llorente A. Lipids in exosomes: current knowledge and the way forward. Prog Lipid Res. 2017;66:30-41. doi:10.1016/j.plipres.2017.03.001

35. Eken C, Martin PJ, Sadallah S, Treves S, Schaller M, Schifferli JA. Ectosomes released by polymorphonuclear neutrophils induce a MerTK-dependent anti-inflammatory pathway in macrophages. $J$ Biol Chem. 2010;285(51):39914-39921. doi:10.1074/jbc. M110.126748

36. MacKenzie A, Wilson HL, Kiss-Toth E, Dower SK, North RA, Surprenant A. Rapid secretion of interleukin-1beta by microvesicle shedding. Immunity. 2001;15(5):825-835.

37. Michaelson DM, Barkai G, Barenholz Y. Asymmetry of lipid organization in cholinergic synaptic vesicle membranes. Biochem J. 1983;211(1):155-162. doi:10.1042/bj2110155

38. Théry C, Boussac M, Véron P, et al. Proteomic analysis of dendritic cell-derived exosomes: a secreted subcellular compartment distinct from apoptotic vesicles. J Immunol. 2001;166(12):7309LP-7318LP. doi:10.4049/jimmunol.166.12.7309

39. Slone EA, Pope MR, Fleming SD. Phospholipid scramblase 1 is required for $\beta 2$-glycoprotein I binding in hypoxia and reoxygenation-induced endothelial inflammation. J Leukoc Biol. 2015;98 (5):791-804. doi:10.1189/jlb.3A1014-480R

40. Sambarey A, Devaprasad A, Baloni P, et al. Meta-analysis of host response networks identifies a common core in tuberculosis. NPJ Syst Biol Appl. 2017;3:4. doi:10.1038/s41540-017-0005-4

41. Greco E, Quintiliani G, Santucci MB, et al. Janus-faced liposomes enhance antimicrobial innate immune response in mycobacterium tuberculosis infection. Proc Natl Acad Sci. 2012;109(21):E1360E1368. doi:10.1073/pnas.1200484109

42. Park S-Y, Jung M-Y, Kim H-J, et al. Rapid cell corpse clearance by stabilin-2, a membrane phosphatidylserine receptor. Cell Death Differ. 2007;15:192. doi:10.1038/sj.cdd.4402242

43. Gasser O, Schifferli JA. Activated polymorphonuclear neutrophils disseminate anti-inflammatory microparticles by ectocytosis. Blood. 2004;104(8):2543-2548. doi:10.1182/blood-2004-01-0361

44. Prados-Rosales R, Baena A, Martinez LR, et al. Mycobacteria release active membrane vesicles that modulate immune responses in a TLR2-dependent manner in mice. J Clin Invest. 2011;121(4):14711483. doi:10.1172/JCI44261 
45. Prados-Rosales R, Carreno LJ, Batista-Gonzalez A, et al. Mycobacterial membrane vesicles administered systemically in mice induce a protective immune response to surface compartments of mycobacterium tuberculosis. MBio. 2014;5:5. doi:10.1128/mBio.01921-14

46. Levin-arama M, Abraham L, Waner T, Harmelin A, Steinberg DM, Lahav T. Subcutaneous compared with intraperitoneal ketamine xylazine for anesthesia of mice. J Am Assoc Lab Anim Sci. 2016;55 (6): 794-800.
47. Tamboli IY, Barth E, Christian L, et al. Statins promote the degradation of extracellular amyloid \{beta\}-peptide by microglia via stimulation of exosome-associated insulin-degrading enzyme (IDE) secretion. J Biol Chem. 2010;285(48):37405-37414. doi:10.1074/ jbc.M110.149468 


\section{Supplementary material}

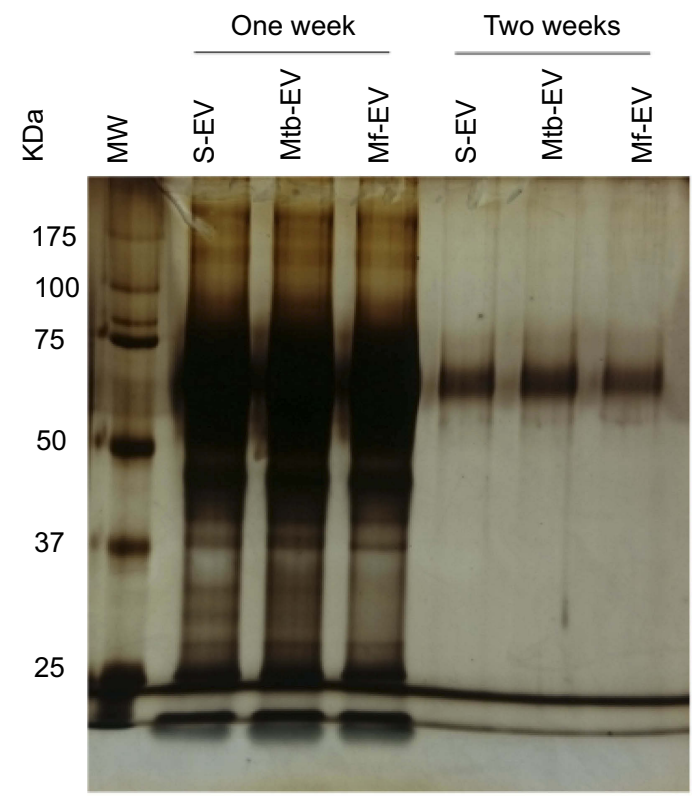

Figure SI EVs are stable at $4^{\circ} \mathrm{C}$ for a week. EVs were purified from J774A.I cells (S-EV), from J774A. I cells that had been infected with $M$. fortuitum for $4 \mathrm{~h}$ (Mf-EV) or from J774A.I cells that had been infected with $M$. tuberculosis $\mathrm{H} 37 \mathrm{Rv}$ for $4 \mathrm{~h}$ (Mtb-EV) and stored at $4{ }^{\circ} \mathrm{C}$ for one or two weeks. The proteins were purified with trichloroacetic acid and loaded into a $5 / 12 \%$ polyacrylamide gel, which was visualized with silver staining.

\section{Publish your work in this journal}

The International Journal of Nanomedicine is an international, peerreviewed journal focusing on the application of nanotechnology in diagnostics, therapeutics, and drug delivery systems throughout the biomedical field. This journal is indexed on PubMed Central, MedLine, CAS, SciSearch ${ }^{\circledR}$, Current Contents ${ }^{\mathbb{B}} /$ Clinical Medicine,
Journal Citation Reports/Science Edition, EMBase, Scopus and the Elsevier Bibliographic databases. The manuscript management system is completely online and includes a very quick and fair peer-review system, which is all easy to use. Visit http://www.dovepress.com/ testimonials.php to read real quotes from published authors. 